\title{
Mechanism of spontaneous hole formation in thin polymeric films
}

\author{
Yu, Kaijia; Rasmussen, Henrik K.; Román Marín, José Manuel; Hassager, Ole
}

Published in:

Physical Review B Condensed Matter

Link to article, DOI:

10.1103/PhysRevB.85.024201

Publication date:

2012

Document Version

Publisher's PDF, also known as Version of record

Link back to DTU Orbit

Citation (APA):

Yu, K., Rasmussen, H. K., Román Marín, J. M., \& Hassager, O. (2012). Mechanism of spontaneous hole formation in thin polymeric films. Physical Review B Condensed Matter, 85, 024201.

https://doi.org/10.1103/PhysRevB.85.024201

\section{General rights}

Copyright and moral rights for the publications made accessible in the public portal are retained by the authors and/or other copyright owners and it is a condition of accessing publications that users recognise and abide by the legal requirements associated with these rights.

- Users may download and print one copy of any publication from the public portal for the purpose of private study or research.

- You may not further distribute the material or use it for any profit-making activity or commercial gain

- You may freely distribute the URL identifying the publication in the public portal

If you believe that this document breaches copyright please contact us providing details, and we will remove access to the work immediately and investigate your claim. 


\title{
Mechanism of spontaneous hole formation in thin polymeric films
}

\author{
Kaijia Yu, ${ }^{1}$ Henrik Koblitz Rasmussen, ${ }^{1}$ Jose Manuel Román Marín, ${ }^{1}$ and Ole Hassager ${ }^{2}$ \\ ${ }^{1}$ Department of Mechanical Engineering, Technical University of Denmark, DK-2800 Kgs. Lyngby, Denmark \\ ${ }^{2}$ Department of Chemical and Biochemical Engineering, Technical University of Denmark, DK-2800 Kgs. Lyngby, Denmark
}

(Received 26 October 2011; published 5 January 2012)

\begin{abstract}
We show computationally that (molten) thin polymeric film containing nonequilibrium configurations originating from a solvent evaporation may develop holes spontaneously in the molten state, and that they appear delayed. Polymers above the glass transition temperature are liquids where the flow depends solely on the nonequilibrium configurations of the molecules.
\end{abstract}

DOI: 10.1103/PhysRevB.85.024201

PACS number(s): $83.80 . \mathrm{Sg}, 47.15 . \mathrm{gm}, 83.80 . \mathrm{Rs}$

\section{INTRODUCTION}

Thin films made by spin coating of diluted polymers followed by solvent evaporation are widely used in material science and technology. Examples are development of new materials ${ }^{1}$ or mouldings of surface topologies ${ }^{2,3}$ where the squeezing of thin polymer films is important for the shaping of well defined micro and nanostructures. The physics behind the dynamics of these molten films remains unanswered. One particular phenomenon is the spontaneous as well as delayed rupture occurring in films heated above the glass transition temperature.

Spontaneous rupture and dewetting of thin polymer films $\left(>20 \mathrm{~nm}\right.$ ) above the glass transition temperature ${ }^{4-6}$ have been discussed in an immense amount of scientific papers but still remains unresolved. This is a surprising phenomenon since intermolecular forces were considered to stabilize a film. ${ }^{7}$ For low molecular weight polymers, the rupture and subsequent dewetting of very thin films, the size of a few nanometers, have actually been simulated and well explained. This is based on the thin film equation, ${ }^{7}$ within intermolecular force theory, as low molecular weight polymers behave as Newtonian liquids. However, these polymers are usually not applied technologically due to their brittle nature in the solid state. As the molecular weight increases, polymers become structured or commonly known as viscoelastic liquids. These liquids flow in complex ways. ${ }^{8,9}$

Inspired by the experimental investigations in Ref. 5, we will here theoretically explain why and show how holes can spontaneously develop in thin films of a viscoelastic polymer melt. As recognized in Ref. 5, it is a consequence of what they referred to as residual stresses in the polymer, originating from fast evaporation of the solvent during the preparation of these films from a polymeric solution. In this paper, our concern is about the film deformation in the initial stages of the hole generation (see Fig. 1) before the hole expands or dewets. Only the hole expansion or dewetting has been discussed in the scientific literature. We will model the flow without the effect of disjoining force until a local film thickness reaches zero [seen in Fig. 1(d)], which by definition is the critical point in time where a hole develops.

\section{COMPUTATIONAL DETAILS}

Polymeric or structured liquids flow as a consequence of nonequilibrium configurations described as stresses. As in most experimental dewetting studies, ${ }^{4,5,10}$ we will focus on the dynamics of monodisperse polymer melts. These melts ideally only contain polymers of one length (e.g., molecular weight). An analytical model (e.g., constitutive equation) for the dynamic of highly entangled monodisperse polymer melts was pioneered by Doi and Edwards, ${ }^{11}$ within the tube ideas of P.G. de Gennes. ${ }^{12}$ While the original Doi and Edwards model ${ }^{11}$ has many limitations, Wagner et al. ${ }^{13}$ added a stretch equation based on the "interchain pressure" concept. ${ }^{14}$ This approach is currently the most accurate model to predict the flow behavior of monodisperse polymer melts. ${ }^{13,15,16}$ The analytical model is

$$
\sigma_{i j}=\int_{-\infty}^{t} M\left(t-t^{\prime}\right) f\left(t, t^{\prime}\right)^{2} 5\left\langle\frac{E_{i n} u_{n} E_{j m} u_{m}}{|\mathbf{E} \cdot \mathbf{u}|^{2}}\right\rangle d t^{\prime},
$$

where

$$
\begin{aligned}
& \frac{\partial}{\partial t} f\left(t, t^{\prime}\right) \\
& \quad=f\left(t, t^{\prime}\right)\left[\frac{\partial}{\partial t}\langle\ln |\mathbf{E} \cdot \mathbf{u}|\rangle-\frac{1}{\tau_{w}} f\left(t, t^{\prime}\right)\left(f\left(t, t^{\prime}\right)^{3}-1\right)\right] \text { and } \\
& f\left(t^{\prime}, t^{\prime}\right)=1,
\end{aligned}
$$

depending on the deformation history. Here the terms $\sigma_{i j}$ are the components of the stress tensor. $f$ is commonly referred to as the molecular stress function. The angular brackets denote an average over a unit sphere $\langle\ldots\rangle=1 /(4 \pi) \int_{|\mathbf{u}|=1} \ldots d \mathbf{u}$. u is a unit vector. The components of the displacement gradient tensor $\mathbf{E}$ is given by $E_{\mathrm{ij}}\left(\boldsymbol{x}, t, t^{\prime}\right)=\partial x_{\mathrm{i}} / \partial x_{\mathrm{j}}{ }^{\prime}, i=1,2,3$ and $j=1,2,3$. $\left(x_{1}^{\prime}, x_{2}^{\prime}, x_{3}^{\prime}\right)$ are the coordinates of a given particle in the stress-free reference state (time $t^{\prime}$ ), displaced to coordinates $\left(x_{1}, x_{2}, x_{3}\right)$ in the current state (time $\left.t\right)$. Here we apply the commonly used Currie approximation ${ }^{17}$ for all terms in the angular brackets as well as an approximation of the molecular stress function. The linear dynamics of the polymer are described by the memory function $M\left(t-t^{\prime}\right)$.

The model above only contains one unknown nonlinear parameter $\tau_{w} . \tau_{w}$ is referred to as the tube diameter relaxation time. There exist only a few experimentally determined (e.g., fitted) values of $\tau_{w}$ for monodisperse polystyrenes. For computational simplicity, we will use an approximation of the molecular stress function $f$ starting from the point where the film melts. The $f$ used in the molten state is the (analytical) solution of the differential equation

$$
\frac{\partial}{\partial t} f\left(t, t^{\prime}\right)=-\frac{1}{\tau_{W}} f\left(t, t^{\prime}\right)^{2}\left(f\left(t, t^{\prime}\right)^{3}-1\right),
$$


with the initial condition $f\left(t^{\prime}, t^{\prime}\right)=\exp (\langle\ln |\mathbf{E} \cdot \mathbf{u}|\rangle)$. A similar approach, although using a simplified molecular stress function of the rouse type, was used to explain the delayed rupture of extended polymer melts. ${ }^{8}$ The above approximation allows us to use the numerical algorithm developed recently by Marín et al. ${ }^{18}$ It is a 3D finite element method where node points follow the particle movement. The method is third order accurate in both space and time, ensuring high computational accuracy.

We will apply the method by Baumgaertel, Schausberger, and Winter $(\mathrm{BSW})^{19}$ to describe the linear dynamics. The memory function is

$$
\begin{gathered}
M\left(t-t^{\prime}\right)=\int_{0}^{\infty} \frac{H(\tau)}{\tau^{2}} e^{\left(-\left(t-t^{\prime}\right) / \tau\right)} d \tau, \\
H(\tau)=n_{e} G_{N}^{0}\left[\left(\frac{\tau}{\tau_{\max }}\right)^{n_{e}}+\left(\frac{\tau}{\tau_{c}}\right)^{-n_{g}}\right] h\left(1-\tau / \tau_{\max }\right)
\end{gathered}
$$

where $h(x)$ is the Heaviside step function. $\tau_{\max }$ is the maximal relaxation time in a continuous distribution of time constant. The plateau modulus $G_{N}^{0}$ corresponds to the natural elasticity of the polymer. $n_{e}, n_{g}, G_{N}^{0}$, and $\tau_{c}$ are material parameters which have a unique value for each type of polymer, whereas $\tau_{\max }$ depends of its molecular weight. ${ }^{20}$

In almost all thin film problems, the surface tension plays a significant role in the dynamics. With increasing film thickness, the intermolecular force theory becomes the continuum mechanical surface (and interface) tension, $\sigma$. For polymers, the surface tension is nondimensionally given as the "surface elasticity" number ${ }^{21}$ as $\phi=\sigma /\left(G_{N}^{0} H_{0}\right)$, relating the surface tension to the plateau modulus. $H_{0}$ is a characteristic dimension of the system, which here is the thickness of the polymeric film. The dynamics of the film is therefore not related to the thickness but nondimensionally to the surface elasticity. As an example, a $60 \mathrm{~nm}$ thick film of polystyrene has a surface elasticity number $\phi$ of about 3 .

A thin polymer film without configurational stress is stable. But polymeric films may contain nonequilibrium configurations originating from the solvent evaporation. ${ }^{4}$ The build of nonequilibrium configurations is a consequence of the reduction of the height of the film as the solvent evaporates. These occur where the dynamics of the polymer chain is slower than the evaporation. The chains are virtually compressed. The dynamics and the configurational changes of the polymeric molecules during the evaporation are highly complex. In the first part of the evaporation, the polymer molecules stay in random configurations, as the dynamics of the polymers in diluted solutions are very fast. As an example, a very high molecular weight $5 \%$ polystyrene solution (about $4000 \mathrm{~kg} / \mathrm{mole}$ ) will have a maximal relaxation time of no more than $1 s$ at room temperature. ${ }^{22}$ At lower molecular weights or higher temperature, this relaxation time will be several decades lower. As evaporation takes place, the polymer concentration increases. Hence, at a certain polymer concentration $(X)$ where the evaporation rate is faster than the dynamics of the polymer chain, the solution will build up nonequilibrium configurations. These will depend on the specific solvent-polymer pair, molecular weight, temperature, and evaporation history. At certain conditions, a film almost without configurational stress may develop. In particular, the temperature and evaporation history is difficult to quantify and has not been reported in any study. Therefore we will introduce specific nonequilibrium configurations of the polymer as the theoretical starting point. For simplicity, we specify the nonequilibrium configurations as the following heuristic approach. A random configured polymer melt is extended biaxially to coat the surface as $\left(x_{1}, x_{2}, x_{3}\right)=\left[x_{1}(0) \cdot \exp (-\dot{\epsilon} \cdot t), x_{2}(0) \cdot \exp (\dot{\epsilon} \cdot t / 2), x_{3}(0) \cdot\right.$ $\exp (\dot{\epsilon} \cdot t / 2)]$ for $t>0$, where the initial particle positions at $t=0$ are given as $\left[x_{1}(0), x_{2}(0), x_{3}(0)\right] . \dot{\epsilon}$ is a constant strain rate and $\epsilon=\dot{\epsilon} \cdot t$ the strain. In this model, we have assumed that a $X \%$ (volume/volume) polymer solution on a surface will be reduced the same amount in all directions during evaporation. Therefore a polymer melt originating from an $X \%$ (volume/volume) solution will need a biaxial extension representing a strain of $\epsilon=-(2 / 3) \ln (X)$ to coat the original surface completely. For example, a $5 \%$ solution represents a strain of $\epsilon=2$.

(a)

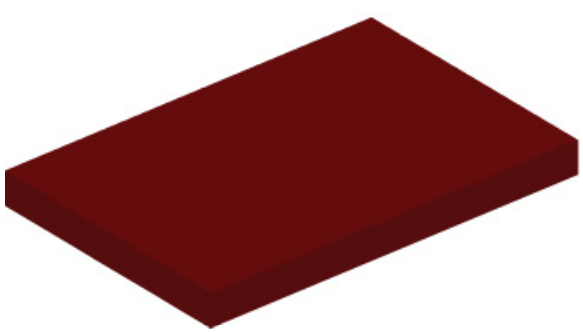

(b)

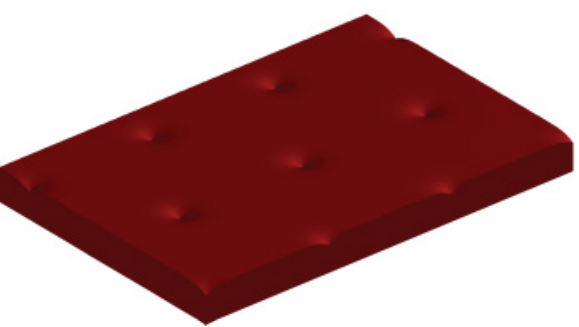

(c)

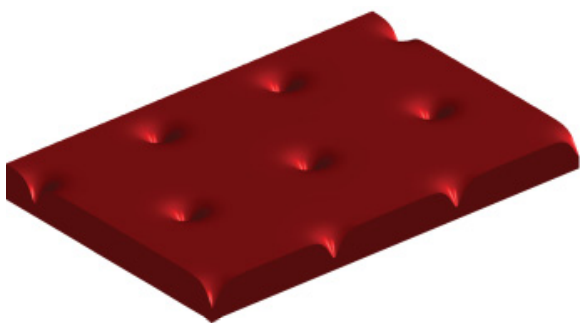

(d)

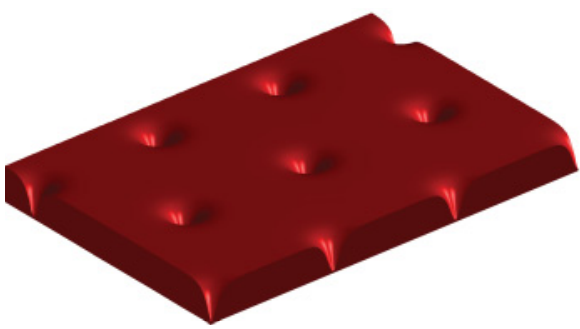

FIG. 1. (Color) The dynamics of the film surface. Notice, the figure is not draw to scale, as the thickness has been increased by a factor of 25. Subfigure (a) shows the initial surface perturbation in a hexagonal pattern, where $S=1.5 \mathrm{~nm}$ and $H_{0}=60 \mathrm{~nm}$. The smallest distance between the holes, relative to the initial thickness, is 100 . 
Within a traditional continuum mechanics framework, the spontaneous development of holes is considered an instability. This instability is highly sensitive to initial inhomogeneities in the film. Computationally we create a perturbation in the ideal flat film by removing a small sinusoidal part of the surface exactly at the end of the initial extension. These depressions are equally spaced in a hexagonal pattern (illustrated in Fig. 1). So the free surface is perturbed just before the film is molten. The sinusoidal shaped perturbation [illustrated in Fig. 1(a)] has a height $S$ and (fixed) radius of $H_{0}$, identical to the film thickness. We exploit the hexagonal symmetry in our computations. As a boundary condition against the substrate, we have used a slip boundary condition. This is an exact boundary condition for the surface dynamics observed in free-standing film. ${ }^{10}$ If the polymer is attached to a nonadsorbing substrate such as PDMS (polydimethylsiloxane), the polymer will experience some resistance in the interface, although experimentally the polymer does not show any resistance of importance against the initial dewetting. ${ }^{5}$ The surface dynamics in free-standing film ${ }^{10}$ are very similar to the dynamics of a film attached to a PDMS substrate. ${ }^{5}$

\section{RESULTS, DISCUSSION AND CONCLUSIONS}

Figures 1(b)-1(d) show the computed time dynamics of the surface. It initially consists of a 60-nm-thick polymeric film of a $390 \mathrm{~kg} /$ mole polystyrene, where we use a value of $\tau_{w} / 3=0.035 \tau_{\max } \cdot{ }^{13,23}$ All material parameters can be found in Wagner et al. ${ }^{13}$ and Bach et al. ${ }^{23}$ After a time delay, the initial disturbance gradually evolves and the local thickness goes toward zero, which by definition is the critical point in time where the hole develops. Only one hole develops from a disturbance, and the surface remains flat between the holes.

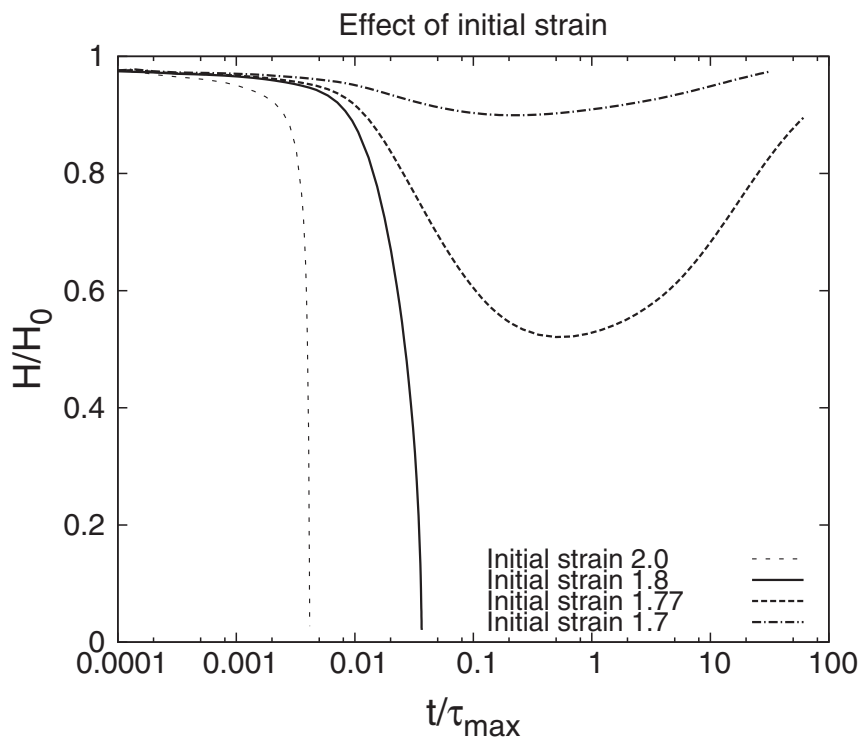

FIG. 2. The smallest film thickness (relative to the initial thickness) as a function of time (relative to the maximal relaxation time $\tau_{\max }$ ). A fixed initial surface perturbation of $S=1.5 \mathrm{~nm}$ (and $H_{0}=60$ $\mathrm{nm}$ ) is used, with a (smallest) distance between the holes, relative to the initial thickness, of 400. The imposed strain values are $\epsilon=2,1.8$, 1.77, and 1.7. The smallest distance between the holes, relative to the initial thickness, is 400 .

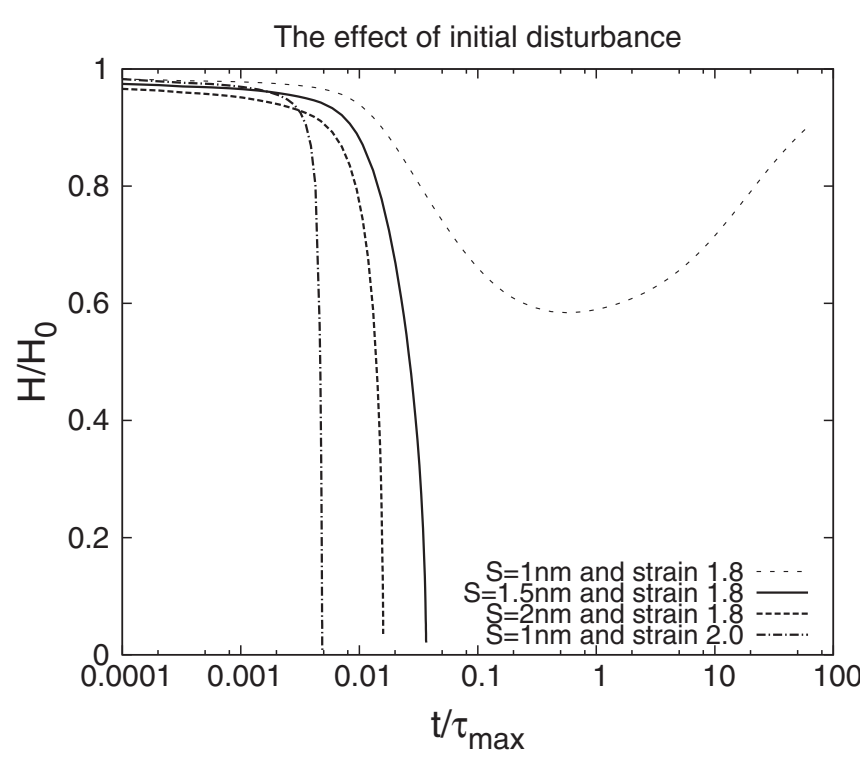

FIG. 3. The smallest film thickness (relative to the initial thickness) as a function of time (relative to the maximal relaxation time $\left.\tau_{\max }\right)$. The initial surface perturbations are $S=2 \mathrm{~nm}, 1.5 \mathrm{~nm}$, and $1 \mathrm{~nm}$. The initial film thickness $H_{0}=60 \mathrm{~nm}$, where the (smallest) distance between the holes, relative to this initial thickness, is 400 . The imposed strain values are $\epsilon=2$ and 1.8 .

This is the surface topology observed in all experimental investigations of the spontaneous hole development in thin polymeric films. ${ }^{5}$ The topology in few-nanometers-thin films of ordinary Newtonian liquids distinguishes from this pattern where the development of one hole from a disturbance is correlated to subsequent surface disturbances which develop into further holes.

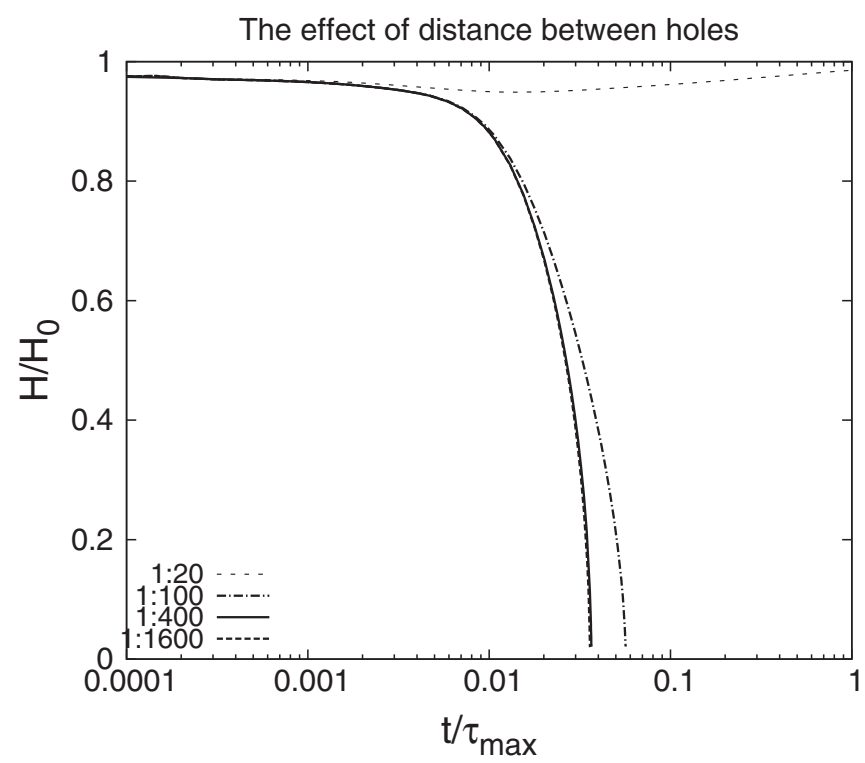

FIG. 4. The smallest film thickness (relative to the initial thickness) as a function of time (relative to the maximal relaxation time $\tau_{\max }$ ). A fixed initial surface perturbation of $S=1.5 \mathrm{~nm}$ (and $H_{0}=60 \mathrm{~nm}$ ) is used, where the imposed strain values is $\epsilon=1.8$. The (smallest) distance between the holes (relative to the initial thickness) are $20,100,400$, and 1600 . 
In Fig. 2, the smallest thickness is shown as a function of time, nondimensionalized by the maximal relaxation time in the polymer $\tau_{\max }$. We have used the same initial surface perturbation and applied a different initial nonequilibrium configuration represented as strain values of $\epsilon=2,1.8,1.77$, and 1.7. At a time considerably smaller than $\tau_{w} / 3$, the polymer is a highly elastic and strain hardening liquid as it experiences interchain pressure $^{14}$ from the extension. The surface is stable in this strain hardening region. At times scales larger than $\tau_{w} / 3$, the polymer flow is in the soft elastic regime. It is in the transition zone between hard and soft elastic response where the holes may develop, and it is experimentally observed as a delay. ${ }^{5}$

As seen in Fig. 2, a strain of $\epsilon=1.8$ represents the smallest amount of nonequilibrium configurations needed for a hole to develop given this specific surface perturbation. At initial strains smaller than this critical strain, the suppression in the surface will gradually disappear at time scales larger than $\tau_{\max }$ where the polymer starts to behave as an ordinary fluid. The initial size of the disturbance is particularly important, as seen in Fig. 3. A too small disturbance will not develop into a hole before the polymer starts to behave as an ordinary fluid. Here the disturbance will vanish. These theoretical findings are in agreement with the observations in Ref. 5, where the number of holes per area was very dependent on the amount of residual stress, reduced by heat treatment in the solid state. The real initial disturbance may be of complex origin, but the hole formation is very sensitive to the size and character of the initial disturbance (see Fig. 3) as well as the initially imposed nonequilibrium configuration. As neither the real initial configurations of the polymers nor the particular spatial distribution of imperfections in the thin film are known quantitatively, a comparison of the theoretical findings with the observed number of holes per area is unfeasible.

The overall topology in thin film ${ }^{5}$ differs from the corresponding observations of few-nanometers-thin film. ${ }^{7}$ Inspecting Fig. 4, it is of interest to notice that the development of holes separated with distances of more than $400 \mathrm{H}_{0}$ seem to be completely unaffected by each other. If the distance is less than $400 H_{0}$, they tend to stabilize each other. This is opposite of the dynamics of development of holes in few-nanometers-thin films of ordinary liquid where nearby disturbances seem to destabilize each other. ${ }^{7}$ A distance of about $200 H_{0}$ represents the smallest distance, between the center of the holes, observed experimentally. ${ }^{5}$ Therefore, the hole development would be expected to be insensitive to the spacial distribution of imperfections in the thin film. Only the initial configurations of the polymers and the particular character of the imperfections seem to be of importance as discussed above in details. To understand why thin films of polymer melt ruptures spontaneously, we need to distinguish between the flow of ordinary and polymeric liquids. Our work gives the background for the prediction of the stability and dynamics of films used in different micro- and nanotechnologies.
${ }^{1}$ P. Podsiadlo, A. K. Kaushik, E. M. Arruda, A. M. Waas, B. S. Shim, J. Xu, H. Nandivada, B. G. Pumplin, J. Lahann, A. Ramamoorthy, and N. A. Kotov, Science 318, 80 (2007).

${ }^{2}$ H. D. Rowland, W. P. King, J. B. Pethica, and G. L. W. Cross, Science 322, 720 (2008).

${ }^{3}$ S. F. Lyuksyutov, R. A. Vaia, P. B. Paramonov, S. Juhl, L. Waterhouse, R. M. Ralich, G. Sigalov, and E. Sancaktar, Nat. Mater. 2, 468 (2003).

${ }^{4}$ G. Reiter and P. G. de Gennes, Eur. Phys. J. E 6, 25 (2001).

${ }^{5}$ G. Reiter, M. Hamieh, P. Damman, S. Sclavons, S. Gabriele,

T. Vilmin, and E. Raphaë, Nat. Mater. 4, 754 (2005).

${ }^{6}$ O. Bäumchen, R. Fetzer, and K. Jacobs, Phys. Rev. Lett. 103, 247801 (2009).

${ }^{7}$ J. Becker, G. Grün, R. Seemann, H. Mantz, K. Jacobs, K. R. Mecke, and R. Blossey, Nat. Mater. 2, 59 (2003).

${ }^{8}$ A. Lyhne, H. K. Rasmussen, and O. Hassager, Phys. Rev. Lett. 102, 138301 (2009).

${ }^{9}$ J. M. Adams and P. D. Olmsted, Phys. Rev. Lett. 102, 067801 (2009).

${ }^{10}$ K. Dalnoki-Veress, B. G. Nickel, C. Roth, and J. R. Dutcher, Phys. Rev. E 59, 2153 (1999).

${ }^{11}$ M. Doi and S. F. Edwards, J. Chem. Soc. Faraday Trans. II 74, 1818 (1978).
${ }^{12}$ P. G. de Gennes, Scaling Concepts in Polymer Physics (Cornell University Press, Ithaca, NY, 1979).

${ }^{13}$ M. H. Wagner, S. Kheirandish, and O. Hassager, J. Rheol. 49, 1317 (2005).

${ }^{14} \mathrm{G}$. Marrucci and G. Ianniruberto, Macromolecules 37, 3934 (2004).

${ }^{15}$ H. K. Rasmussen, A. L. Skov, J. K. Nielsen, and P. Laillé, J. Rheol. 53, 401 (2009).

${ }^{16}$ M. H. Wagner and V. H. Rolón-Garrido, Rheol. Acta 49, 459 (2010).

${ }^{17}$ P. K. Currie, J. Non-Newtonian Fluid Mech. 11, 53 (1982).

${ }^{18}$ J. M. R. Marín and H. K. Rasmussen, J. Non-Newtonian Fluid Mech. 156, 177 (2009).

${ }^{19}$ M. Baumgaertel, A. Schausberger, and H. H. Winter, Rheol. Acta 29, 400 (1990).

${ }^{20}$ M. Doi and S. F. Edwards, The Theory of Polymer Dynamics (Clarendon Press, Oxford, 1986).

${ }^{21}$ H. K. Rasmussen and O. Hassager, J. Rheol. 45, 527 (2001).

${ }^{22}$ P. Attane, P. LeRoy, J. M. Pierrard, and G. Turrel, J. Non-Newtonian Fluid Mech. 9, 13 (1981).

${ }^{23}$ A. Bach, K. Almdal, H. K. Rasmussen, and O. Hassager, Macromolecules 36, 5174 (2003). 